\title{
Actinomycotic Pituitary Abscess: Unusual Pathology, Unexpected Organism
}

Sir,

A 46-year-old female presented with complaints of progressively worsening headache and visual deterioration of 2 weeks' duration. On examination, she was found to have bitemporal hemianopia. Magnetic resonance imaging (MRI) showed a sellar-suprasellar cystic mass extending up to the optic chiasm. The mass was hyperintense on $\mathrm{T} 1$ and isointense on $\mathrm{T} 2$ sequences with a hypointense rim [Figure 1]. Pituitary adenoma with previous apoplexy was suspected. A transsphenoidal approach to excise the lesion was undertaken, but on opening the capsule yellowish pus came out. This was sent for microbiological examination and a part of the capsule wall was sent for histology. There was no growth on culture and neither could Gram stain demonstrate any organism. However, histopathological examination showed the typical morphology of a sulfur granule with periodic acidSchiff-positive short rod-like, filamentous structures radiating outward. An eosinophilic, amorphous material was seen around the pathogenic organisms - SplendoreHoeppli phenomenon [Figure 2]. A diagnosis of actinomycotic abscess of the pituitary gland was made. The patient had an uneventful postoperative recovery with improvement of vision and decrease of headache. Initially, she was treated with injection ceftriaxone for 2 weeks, but later this was switched to oral amoxicillin and cloxacillin and this was continued for 4 months. Postoperative MRI done after 1 year showed no residual lesion [Figure 3].

Actinomyces are facultatively anaerobic, Gram-positive bacteria $^{[1,2]}$ which were once confused with fungi. They 
are present as normal commensals in the mucosa of the oropharynx and the alimentary tract. ${ }^{[1]}$ Intracranial involvement though rare is usually in the form of intracerebral subdural and epidural abscesses as well as meningitis. ${ }^{[1]}$ In our case, it had caused a pituitary abscess and we could find only one similar report in the literature. ${ }^{[2]}$

Various authors have stressed that the preoperative diagnosis of pituitary abscess is difficult ${ }^{[2,3]}$ due to both their rarity and lack of any differentiating clinical features from tumor masses in this region. As in our case, headache and visual deficits are the most common presenting features. Like in this case, signs of infection are often absent. ${ }^{[3]}$ Imaging too is nonspecific and common differentials of a cystic lesion in this region would include pituitary apoplexy, cystic craniopharyngiomas, and Rathke's cleft cysts. ${ }^{[4]}$

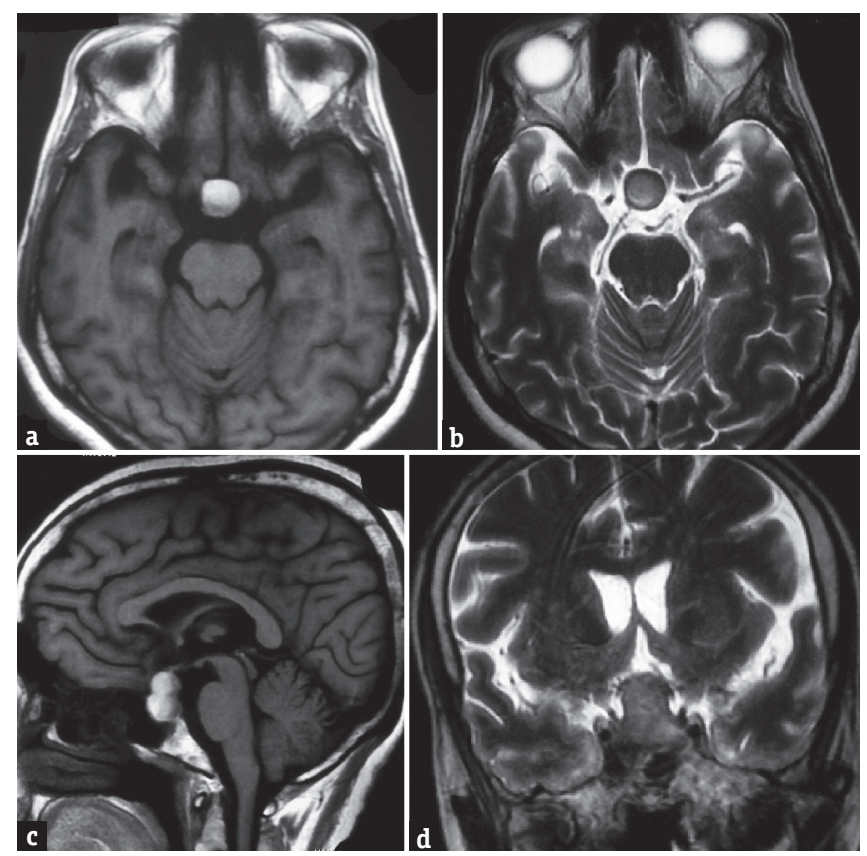

Figure 1: Axial magnetic resonance images showing (a) T1 hyperintense and (b) T2 isointense mass with a hypointense rim in the suprasellar region. Sagittal T1 image (c) showing the pituitary stalk is lifted up by the sellar-suprasellar mass with a fluid level inside and (d) coronal images showing the mass is reaching the optic chiasma
Rahiminejad et al., ${ }^{[1]}$ however, state that the presence of a T2 hypointense rim as was seen in our case is suggestive of actinomycotic abscess.

Pituitary abscesses have been classified as primary or secondary based on whether the abscess occurred in a previously healthy gland or occurred secondary to bacterial seeding in a preexisting pituitary lesion. ${ }^{[2,4]}$ As no previous imaging was present in this instance, it is not possible for us to classify this case in this manner.

In several pituitary abscesses, the causative organism is not unmasked. ${ }^{[4]}$ We too did not initially get any organism on Gram stain or culture. Only a routine aerobic culture of the pus had been sent with no special provisions for the growth of any anaerobic organism. The histopathology report from the piece of wall sent came as a surprise and forced us to revise both the antibiotics used and duration. Actinomyces spp. are sensitive to amoxicillin and this has also been described as a "preferred treatment option." ${ }^{[2]}$ In our case, we continued the same for 4 months. The source of the infection remains unclear in our case. While both hematogenous spread and extension from contiguous sites $^{[5]}$ have been described in the genesis of pituitary abscesses, as our patient had no systemic symptoms and since Actinomyces is a known oropharyngeal commensal, we are tempted to consider spread from the local area as the cause of this pathology.

In conclusion, pituitary abscess is an uncommon disease which mimics tumors in this area in its mode of presentation. If pus is encountered, a hunt must also be made for uncommon organisms such as anaerobes, fungi, and mycobacteria in addition to routine aerobic cultures and staining. Finally, despite drainage of pus, some bit of wall must always be sent for histopathology as this may unmask organisms not detected by microbiological examination.

\section{Declaration of patient consent}

The authors certify that they have obtained all appropriate patient consent forms. In the form the

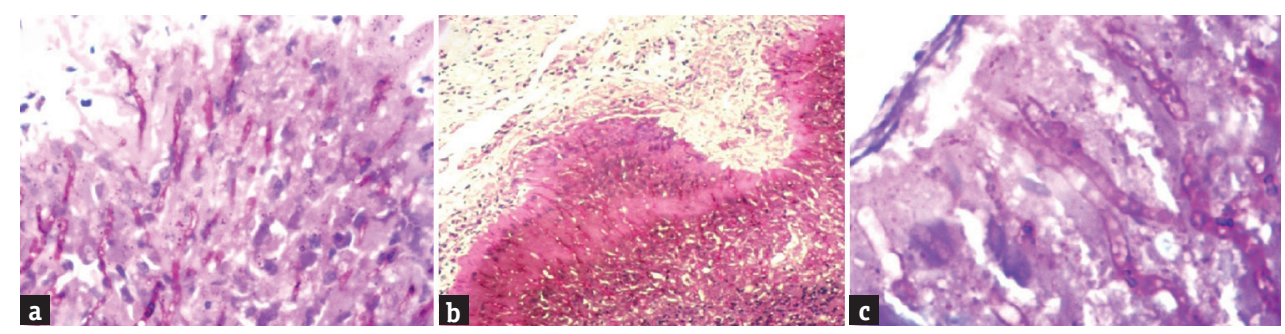

Figure 2: Histopathological images showing (a) periodic acid-Schiff-positive filamentous structures which are (b) radiating outward on an eosinophilic background. A magnified view (c) of the magenta-colored filamentous rods is shown 

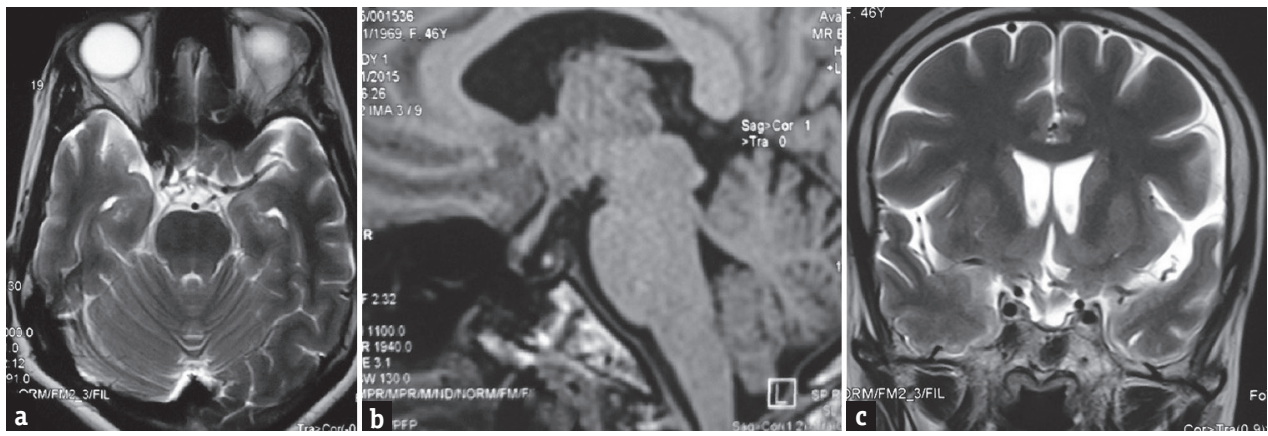

Figure 3: Postoperative magnetic resonance images (a) T2 axial, (b) T1 sagittal, and (c) T2 coronal showing no residual lesion. The hyperintense speck of the posterior pituitary is seen in the sagittal image inside the sella

patient(s) has/have given his/her/their consent for his/ her/their images and other clinical information to be reported in the journal. The patients understand that their names and initials will not be published and due efforts will be made to conceal their identity, but anonymity cannot be guaranteed.

\section{Financial support and sponsorship}

Nil.

\section{Conflicts of interest}

There are no conflicts of interest.

Sachinkumar Maheshbhai Patel, Sugat Sanyal, Prasad Krishnan, Kanchan Kumar Mukherjee ${ }^{2}$

Department of Neurosurgery, National Neurosciences Centre, ${ }^{1}$ Department of Pathology, Peerless Hospital and

B K Roy Research Centre, Kolkata, West Bengal, ${ }^{2}$ Department of Neurosurgery, Postgraduate Institute of Medical Education and Research, Chandigarh, India

Address for correspondence: Dr. Prasad Krishnan, Department of Neurosurgery, National Neurosciences Centre, Peerless Hospital Campus, $2^{\text {nd }}$ Floor, 360 Panchasayar, Kolkata - 700 094, West Bengal, India. E-mail: prasad.krishnan@rediffmail.com

\section{REFERENCES}

1. Rahiminejad M, Hasegawa $H$, Papadopoulos $M$, MacKinnon A. Actinomycotic brain abscess. BJR Case Rep 2016;2:20150370.
2. Yapar B, Ozdemir K, Kutlu M, Civlan S, Kocaoglu M, Baykara E, et al. A rare sellar lesion: Pituitary actinomyces infection. J Brain Spinal Cancer 2015;1:15-8.

3. Liu J, You C. Primary pituitary abscess. Neurol India 2015;63:1000-1.

4. Karagiannis AK, Dimitropoulou F, Papatheodorou A, Lyra S, Seretis A, Vryonidou A, et al. Pituitary abscess: A case report and review of the literature. Endocrinol Diabetes Metab Case Rep 2016;2016:160014.

5. Akhaddar A, Elouennass $M$, Baallal $H$, Boucetta $M$. Focal intracranial infections due to actinomyces species in immunocompetent patients: Diagnostic and therapeutic challenges. World Neurosurg 2010;74:346-50.

This is an open access journal, and articles are distributed under the terms of the Creative Commons Attribution-NonCommercial-ShareAlike 4.0 License, which allows others to remix, tweak, and build upon the work non-commercially, as long as appropriate credit is given and the new creations are licensed under the identical terms.

\begin{tabular}{|l|l|}
\hline \multicolumn{2}{|c|}{ Access this article online } \\
\hline Quick Response Code: & Website: \\
\hline
\end{tabular}

How to cite this article: Patel SM, Sanyal S, Krishnan P, Mukherjee KK. Actinomycotic pituitary abscess: Unusual pathology, unexpected organism. J Neurosci Rural Pract 2018;9:441-3.

(c) 2018 Journal of Neurosciences in Rural Practice | Published by Wolters Kluwer - Medknow 